\title{
Dumont's statistic on words
}

\author{
Mark Skandera \\ Department of Mathematics \\ University of Michigan, Ann Arbor, MI \\ mskan@math.lsa.umich.edu \\ Submitted: August 4, 2000; Accepted: January 15, 2001. \\ MR Subject Classifications: 06A07, 68R15
}

\begin{abstract}
We define Dumont's statistic on the symmetric group $S_{n}$ to be the function dmc: $S_{n} \rightarrow \mathbb{N}$ which maps a permutation $\sigma$ to the number of distinct nonzero letters in $\operatorname{code}(\sigma)$. Dumont showed that this statistic is Eulerian. Naturally extending Dumont's statistic to the rearrangement classes of arbitrary words, we create a generalized statistic which is again Eulerian. As a consequence, we show that for each distributive lattice $J(P)$ which is a product of chains, there is a poset $Q$ such that the $f$-vector of $Q$ is the $h$-vector of $J(P)$. This strengthens for products of chains a result of Stanley concerning the flag $h$-vectors of Cohen-Macaulay complexes. We conjecture that the result holds for all finite distributive lattices.
\end{abstract}

\section{Introduction}

Let $S_{n}$ be the symmetric group on $n$ letters, and let us write each permutation $\pi$ in $S_{n}$ in one line notation: $\pi=\pi_{1} \cdots \pi_{n}$. We call position $i$ a descent in $\pi$ if $\pi_{i}>\pi_{i+1}$, and an excedance in $\pi$ if $\pi_{i}>i$. Counting descents and excedances, we define two permutation statistics des : $S_{n} \rightarrow \mathbb{N}$ and exc $: S_{n} \rightarrow \mathbb{N}$ by

$$
\begin{aligned}
& \operatorname{des}(\pi)=\#\left\{i \mid \pi_{i}>\pi_{i+1}\right\} \\
& \operatorname{exc}(\pi)=\#\left\{i \mid \pi_{i}>i\right\}
\end{aligned}
$$

It is well known that the number of permutations in $S_{n}$ with $k$ descents equals the number of permutations in $S_{n}$ with $k$ excedances. This number is often denoted $A(n, k+1)$ and the generating function

$$
A_{n}(x)=\sum_{k=0}^{n-1} A(n, k+1) x^{k+1}=\sum_{\pi \in S_{n}} x^{1+\operatorname{des}(\pi)}=\sum_{\pi \in S_{n}} x^{1+\operatorname{exc}(\pi)}
$$


is called the $n$th Eulerian polynomial. Any permutation statistic stat : $S_{n} \rightarrow \mathbb{N}$ satisfying

$$
A_{n}(x)=\sum_{\pi \in S_{n}} x^{1+\operatorname{stat}(\pi)}
$$

or equivalently,

$$
\#\left\{\pi \in S_{n} \mid \operatorname{stat}(\pi)=k\right\}=\#\left\{\pi \in S_{n} \mid \operatorname{des}(\pi)=k\right\}, \text { for } k=0, \ldots, n-1
$$

is called Eulerian.

A third Eulerian statistic, essentially defined by Dumont [6], counts the number of distinct nonzero letters in the code of a permutation. We define $\operatorname{code}(\pi)$ to be the word $c_{1} \cdots c_{n}$, where

$$
c_{i}=\#\left\{j>i \mid \pi_{j}<\pi_{i}\right\} .
$$

Denoting Dumont's statistic by $d m c$, we have

$$
\operatorname{dmc}(\pi)=\#\{\ell \neq 0 \mid \ell \text { appears in } \operatorname{code}(\pi)\} \text {. }
$$

\section{Example 1.1.}

$$
\begin{aligned}
\pi & =284367951 \\
\operatorname{code}(\pi) & =162122210 .
\end{aligned}
$$

The distinct nonzero letters in $\operatorname{code}(\pi)$ are $\{1,2,6\}$. Thus, $\operatorname{dmc}(\pi)=3$.

Dumont showed bijectively that the statistic dmc is Eulerian. While few researchers have found an application for Dumont's statistic since [6], Foata [8] proved the following equidistribution result involving the statistics INV (inversions) and MAJ (major index). These two statistics belong to the class of Mahonian statistics. (See [8] for further information.)

Theorem 1.1. The Eulerian-Mahonian statistic pairs (des, INV) and (dmc, MAJ) are equally distributed on $S_{n}$, i.e.

$$
\#\left\{\pi \in S_{n} \mid \operatorname{des}(\pi)=k ; \operatorname{INV}(\pi)=p\right\}=\#\left\{\pi \in S_{n} \mid \operatorname{dmc}(\pi)=k ; \operatorname{MAJ}(\pi)=p\right\} .
$$

Note that the statistics des, exc, and dmc are defined in terms of set cardinalities. We denote the descent set and excedance set of a permutation $\pi$ by $D(\pi)$ and $E(\pi)$, respectively. We define the letter set of an arbitrary word $w$ to be the set of its nonzero letters, and denote this by $L(w)$. We will denote the letter set of $\operatorname{code}(\pi)$ by $L C(\pi)$. Thus,

$$
\begin{aligned}
\operatorname{des}(\pi) & =|D(\pi)|, \\
\operatorname{exc}(\pi) & =|E(\pi)|, \\
\operatorname{dmc}(\pi) & =|L C(\pi)| .
\end{aligned}
$$


It is easy to see that for every subset $T$ of $[n-1]=\{1, \ldots, n-1\}$, there are permutations $\pi, \sigma$, and $\rho$ in $S_{n}$ satisfying

$$
T=D(\pi)=E(\sigma)=L C(\rho) .
$$

In fact, Dumont's original bijection [6] shows that for each such subset $T$ we have

$$
\#\left\{\pi \in S_{n} \mid E(\pi)=T\right\}=\#\left\{\pi \in S_{n} \mid L C(\pi)=T\right\} .
$$

However, the analogous statement involving $D(\pi)$ is not true.

Generalizing permutations on $n$ letters are words $w=w_{1} \cdots w_{m}$ on $n$ letters, where $m \geq n$. We will assume that each letter in $[n]$ appears at least once in $w$. Generalizing the symmetric group $S_{n}$, we define the rearrangement class of $w$ by

$$
R(w)=\left\{w_{\sigma^{-1}(1)} \cdots w_{\sigma^{-1}(m)} \mid \sigma \in S_{m}\right\} .
$$

Each element of $R(w)$ is called a rearrangement of $w$.

Many definitions pertaining to $S_{n}$ generalize immediately to the rearrangement class of any word. In particular, the definitions of descent, descent set, code, letter set of a code, and Dumont's statistic remain the same for words as for permutations. Generalization of excedances requires only a bit of effort.

For any word $w$, denote by $\bar{w}=\bar{w}_{1} \cdots \bar{w}_{m}$ the unique nondecreasing rearrangement of $w$. We define position $i$ to be an excedance in $w$ if $w_{i}>\bar{w}_{i}$. Thus,

$$
\operatorname{exc}(w)=\#\left\{i \mid w_{i}>\bar{w}_{i}\right\}
$$

If position $i$ is an excedance in word $w$, we will refer to the letter $w_{i}$ as the value of excedance $i$. One can see word excedances most easily by associating to the word $w$ the biword

$$
\left(\begin{array}{l}
\bar{w} \\
w
\end{array}\right)=\left(\begin{array}{l}
\bar{w}_{1} \cdots \bar{w}_{m} \\
w_{1} \cdots w_{m}
\end{array}\right)
$$

Example 1.2. Let $w=312312311$. Then,

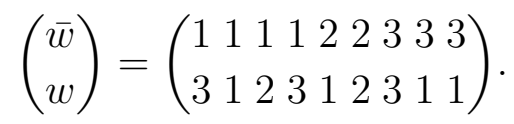

Thus, $E(w)=\{1,3,4\}$ and $\operatorname{exc}(w)=3$. The corresponding excedance values are 3,2 , and 3 .

We will use biwords not only to expose excedances, but to define and justify maps in Sections 3 and 4. In particular, if $u=u_{1} \cdots u_{m}$ and $v=v_{1} \cdots v_{m}$ are words and $y$ is the biword

$$
y=\left(\begin{array}{l}
u \\
v
\end{array}\right)
$$

THE ELECTRONIC JOURNAL OF COMBINATORICS 8 (2001), \#R11 
then we will define biletters $y_{1}, \ldots, y_{m}$ by

$$
y_{i}=\left(\begin{array}{l}
u_{i} \\
v_{i}
\end{array}\right),
$$

and will define the rearrangement class of $y$ by

$$
R(y)=\left\{y_{\sigma^{-1}(1)} \cdots y_{\sigma^{-1}(m)} \mid \sigma \in S_{m}\right\} .
$$

A well known result concerning word statistics is that the statistics des and exc are equally distributed on the rearrangement class of any word $w$,

$$
\#\{y \in R(w) \mid \operatorname{exc}(y)=k\}=\#\{y \in R(w) \mid \operatorname{des}(y)=k\} .
$$

Analogously to the case of permutation statistics, a word statistic stat is called Eulerian if it satisfies

$$
\#\{y \in R(w) \mid \operatorname{stat}(y)=k\}=\#\{y \in R(w) \mid \operatorname{des}(y)=k\}
$$

for any word $w$ and any nonnegative integer $k$.

In Section 2, we state and prove our main result: that dmc is Eulerian as a word statistic. Our bijection is different than that of Dumont [6], which doesn't generalize obviously to the case of arbitrary words. Applying the main theorem to a problem involving $f$-vectors and $h$-vectors of partially ordered sets, we state a second theorem in Section 3. This result strengthens a special case of a result of Stanley [9] concerning the flag $h$-vectors of balanced Cohen-Macaulay complexes. We prove the second theorem in Sections 4 and 5, and finish with some related open questions in Section 6.

\section{Main theorem}

As implied in Section 1, we define Dumont's statistic on an arbitrary word $w$ to be the number of distinct nonzero letters in $\operatorname{code}(w)$.

$$
\operatorname{dmc}(w)=|L C(w)| \text {. }
$$

This generalized statistic is Eulerian.

Theorem 2.1. If $R(w)$ is the rearrangement class of an arbitrary word $w$ and $k$ is any nonnegative integer, then

$$
\#\{v \in R(w) \mid \operatorname{dmc}(v)=k\}=\#\{v \in R(w) \mid \operatorname{exc}(v)=k\} .
$$

Our bijective proof of the theorem depends upon an encoding of a word which we call the excedance table.

Definition 2.1. Let $v=v_{1} \cdots v_{m}$ be an arbitrary word and let $c=c_{1} \cdots c_{m}$ be its code. Define the excedance table of $v$ to be the unique word etab $(v)=e_{1} \cdots e_{m}$ satisfying 
1. If $i$ is an excedance in $v$, then $e_{i}=i$.

2. If $c_{i}=0$, then $e_{i}=0$.

3. Otherwise, $e_{i}$ is the $c_{i}$ th excedance of $v$ having value at least $v_{i}$.

Note that $\operatorname{etab}(v)$ is well defined for any word $v$. In particular, if $i$ is not an excedance in $v$ and if $c_{i}>0$, then there are at least $c_{i}$ excedances in $v$ having value at least $v_{i}$. To see this, define

$$
k=\#\left\{j \in[m] \mid v_{j}<v_{i}\right\}
$$

Since $c_{i}$ of the letters $\bar{v}_{1}, \ldots, \bar{v}_{k}$ appear to the right of position $i$ in $v$, then at least $c_{i}$ of the letters $\bar{v}_{k+1}, \ldots, \bar{v}_{m}$ must appear in the first $k$ positions of $v$. The positions of these letters are necessarily excedances in $v$. An important property of the excedance table is that the letter set of $\operatorname{etab}(v)$ is precisely the excedance set of $v$.

Example 2.2. Let $v=514514532$, and define $c=\operatorname{code}(v)$. Using $v, \bar{v}$, and $c$, we calculate $e=\operatorname{etab}(v)$

$$
\begin{aligned}
& \bar{v}=112344555, \\
& v=514514532, \\
& c=603402210, \\
& e=103403410 .
\end{aligned}
$$

Calculation of $e_{1}, \ldots, e_{5}$ and $e_{9}$ is straightforward since the positions $i=1, \ldots, 5$ and 9 are excedances in $v$ or satisfy $c_{i}=0$. We calculate $e_{6}, e_{7}$, and $e_{8}$ as follows. Since $c_{6}=2$, and the second excedance in $v$ with value at least $v_{6}=4$ is 3 , we set $e_{6}=3$. Since $c_{7}=2$, and the second excedance in $v$ with value at least $v_{7}=5$ is 4 , we set $e_{7}=4$. Since $c_{8}=1$, and the first excedance in $v$ with value at least $v_{8}=3$ is 1 , we set $e_{8}=1$.

We prove Theorem 2.1 with a bijection $\theta: R(w) \rightarrow R(w)$ which satisfies

$$
E(v)=L C(\theta(v))
$$

and therefore

$$
\operatorname{exc}(v)=\operatorname{dmc}(\theta(v))
$$

Definition 2.3. Let $w=w_{1} \cdots w_{m}$ be any word. Define the map $\theta: R(w) \rightarrow R(w)$ by applying the following procedure to an arbitrary element $v$ of $R(w)$.

1. Define the biword $z=\left(\begin{array}{c}v \\ \operatorname{etab}(v)\end{array}\right)$.

2. Let $y$ be the unique rearrangement of $z$ satisfying $y=\left(\begin{array}{c}u \\ \operatorname{code}(u)\end{array}\right)$.

3. Set $\theta(v)=u$. 
Construction of $y$ is quite straightforward. Let $e=e_{1} \cdots e_{m}=\operatorname{etab}(v)$, and linearly order the biletters $z_{1}, \ldots, z_{m}$ by setting $z_{i}<z_{j}$ if

$$
\begin{aligned}
& v_{i}<v_{j}, \text { or } \\
& v_{i}=v_{j} \text { and } e_{i}>e_{j} .
\end{aligned}
$$

Break ties arbitrarily. Considering the biletters according to this order, insert each biletter $z_{i}$ into $y$ to the left of $e_{i}$ previously inserted biletters.

Example 2.4. Let $v$ and $e$ be as in Example 2.2. To compute $\theta(v)$, we define

$$
z=\left(\begin{array}{l}
v \\
e
\end{array}\right)=\left(\begin{array}{lllllllll}
5 & 1 & 4 & 5 & 1 & 4 & 5 & 3 & 2 \\
1 & 0 & 3 & 4 & 0 & 3 & 4 & 1 & 0
\end{array}\right) .
$$

We consider the biletters of $z$ in the order

$$
\left(\begin{array}{l}
1 \\
0
\end{array}\right),\left(\begin{array}{l}
1 \\
0
\end{array}\right),\left(\begin{array}{l}
2 \\
0
\end{array}\right),\left(\begin{array}{l}
3 \\
1
\end{array}\right),\left(\begin{array}{l}
4 \\
3
\end{array}\right),\left(\begin{array}{l}
4 \\
3
\end{array}\right),\left(\begin{array}{l}
5 \\
4
\end{array}\right),\left(\begin{array}{l}
5 \\
4
\end{array}\right),\left(\begin{array}{l}
5 \\
1
\end{array}\right),
$$

and insert them individually into $y$ :

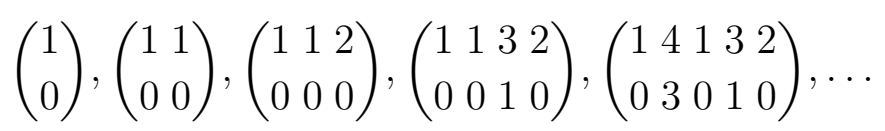

Finally we obtain

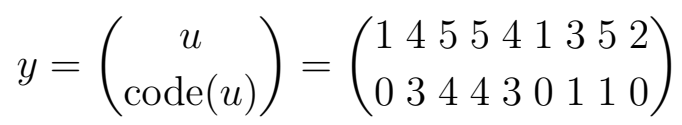

and set $\theta(v)=145541352$.

It is easy to see that any biword $z$ has at most one rearrangement $y$ satisfying Definition 2.3 (2). Such a rearrangement exists if and only if we have

$$
e_{i} \leq \#\left\{j \in[m] \mid v_{j}<v_{i}\right\}, \text { for } i=1, \ldots, m,
$$

or equivalently, if and only if

$$
\bar{v}_{e_{i}}<v_{i} \text {, for } i=1, \ldots, m \text {, }
$$

where we define $\bar{v}_{0}=0$ for convenience.

Observation 2.2. Let $v=v_{1} \cdots v_{m}$ be any word and let $e=\operatorname{etab}(v)$. Then we have

$$
e_{i} \leq \#\left\{j \in[m] \mid v_{j}<v_{i}\right\}, \text { for } i=1, \ldots, m .
$$


Proof. If $i$ is an excedance in $v$, then $e_{i}=i$ and $\bar{v}_{1} \leq \cdots \leq \bar{v}_{i}<v_{i}$. If $c_{i}=0$, then $e_{i}=0$. Otherwise, define

$$
k=\#\left\{j \in[m] \mid v_{j}<v_{i}\right\} .
$$

By the discussion following Definition 2.1, at least $c_{i}$ of the positions $1, \ldots, k$ are excedances in $v$ with values at least $v_{i}$. The letter $e_{i}$, being one of these excedances, is therefore at most $k$.

Thus the map $\theta$ is well defined and satisfies (2.1) and (2.2). We invert $\theta$ by applying the procedure in the following proposition.

Proposition 2.3. Let $y=\left(\begin{array}{l}u \\ c\end{array}\right)=\left(\begin{array}{l}u_{1} \cdots u_{m} \\ c_{1} \cdots c_{m}\end{array}\right)$ be a biword satisfying $c=\operatorname{code}(u)$. The following procedure produces a rearrangement $z=\left(\begin{array}{l}v \\ e\end{array}\right)$ of y satisfying $e=\operatorname{etab}(v)$.

1. For each letter $\ell$ in $L(c)$, find the greatest index $i$ satisfying $c_{i}=\ell$, and define $z_{\ell}=y_{i}$. Let $S$ be the set of such greatest indices, let $T=[m] \backslash S$, and let $t=|T|$.

2. For each index $i \in T$, define

$$
d_{i}= \begin{cases}\#\left\{j \in S \mid c_{j} \leq c_{i} ; u_{j} \geq u_{i}\right\}, & \text { if } c_{i}>0 \\ 0, & \text { otherwise }\end{cases}
$$

3. Define a map $\sigma: T \rightarrow[t]$ such that $y_{\sigma^{-1}(1)} \cdots y_{\sigma^{-1}(t)}$ is the unique rearrangement of $\left(y_{i}\right)_{i \in T}$ satisfying

$$
d_{\sigma^{-1}(1)} \cdots d_{\sigma^{-1}(t)}=\operatorname{code}\left(u_{\sigma^{-1}(1)} \cdots u_{\sigma^{-1}(t)}\right) .
$$

4. Insert the biletters $y_{\sigma^{-1}(1)} \cdots y_{\sigma^{-1}(t)}$ in order into the remaining positions of $z$.

Proof. The procedure above is well defined. In particular, we may perform step 3 because the biword $\left(\begin{array}{l}u_{i} \\ d_{i}\end{array}\right)_{i \in T}$ satisfies

$$
d_{i} \leq \#\left\{j \in T \mid u_{j}<u_{i}\right\}, \text { for each } i \in T,
$$

as required by (2.3). To see that this is the case, let $i$ be an index in $T$ with $c_{i}>0$. In step 1 we have placed $d_{i}$ biletters $y_{j}$ with $u_{j} \geq u_{i}>\bar{u}_{c_{i}}$ into positions $1, \ldots, c_{i}$ of $z$. Thus, at least $d_{i}$ biletters $y_{j}$ with $u_{j} \leq \bar{u}_{c_{i}}$ have not been placed into these positions. The index $j$ of any such biletter belongs to $S$ only if $c_{j}>c_{i}$. However, since $\bar{u}_{c_{j}}<u_{j} \leq \bar{u}_{c_{i}}<u_{i}$, we have $c_{j}<c_{i}$. Thus, $j$ belongs to $T$.

To prove that the biword $z=\left(\begin{array}{l}v \\ e\end{array}\right)$ produced by our procedure satisfies $e=\operatorname{etab}(v)$, we will calculate the excedance set of $v$ and will verify that $e$ satisfies the conditions of Definition 2.1.

First we claim that $E(v)=L(c)$. Certainly the positions $L(c)=\left\{c_{j} \mid j \in S\right\}$ are excedances in $v$, because for each index $j$ in $S$, we have $v_{c_{j}}=u_{j}>\bar{u}_{c_{j}}=\bar{v}_{c_{j}}$. Thus, $L(c) \subset E(v)$. Suppose that the reverse inclusion is not true. For each index $j$ in $T$, 
denote by $\phi(j)$ the position of $z$ into which we have placed $y_{j}$. Assuming that some indices $\{\phi(j) \mid j \in T\}$ are excedances in $v$, choose $i \in T$ so that $\phi(i)$ is the leftmost of these excedances. Let $k$ be the number of positions of $u$ holding letters strictly less than $u_{i}$,

$$
k=\#\left\{j \in[m] \mid u_{j}<u_{i}\right\} .
$$

Since $\phi(i)$ is an excedance in $v$, the subword $z_{1} \cdots z_{k}$ of $z$ contains the biletter $y_{i}$, all biletters $\left\{y_{j} \mid j \in T, \phi(i)<\phi(j)\right\}$, and all biletters $\left\{y_{j} \mid j \in S, c_{j} \leq k\right\}$. Thus,

$$
k>\#\left\{j \in S \mid c_{j} \leq k\right\}+\#\{j \in T \mid \phi(j)<\phi(i)\} .
$$

Since $c_{i} \leq k$ by $(2.3)$, we may rewrite $\#\left\{j \in S \mid c_{j} \leq k\right\}$ as

$$
\#\left\{j \in S \mid c_{j} \leq k\right\}=\#\left\{j \in S \mid c_{j} \leq c_{i}\right\}+\#\left\{j \in S \mid c_{i}<c_{j} \leq k\right\} .
$$

Using the definition of $\sigma$ and noting that $\sigma(j)<\sigma(i)$ implies $u_{j}<u_{i}$, we may rewrite $\#\{j \in T \mid \phi(j)<\phi(i)\}$ as

$$
\begin{aligned}
\#\{j \in T \mid \phi(j)<\phi(i)\} & =\#\{j \in T \mid \sigma(j)<\sigma(i)\} \\
& =\#\left\{j \in T \mid u_{j}<u_{i}\right\}-\#\left\{j \in T \mid u_{j}<u_{i} ; \sigma(j)>\sigma(i)\right\} \\
& =\#\left\{j \in T \mid u_{j}<u_{i}\right\}-\left(\sigma(i) \text { th letter of } \operatorname{code}\left(u_{\sigma^{-1}(1)} \cdots u_{\sigma^{-1}(t)}\right)\right) \\
& =\#\left\{j \in T \mid u_{j}<u_{i}\right\}-d_{i} \\
& =\#\left\{j \in T \mid u_{j}<u_{i}\right\}-\#\left\{j \in S \mid c_{j} \leq c_{i} ; u_{j} \geq u_{i}\right\} .
\end{aligned}
$$

Applying these identities to (2.5), we obtain

$$
\#\left\{j \in S \mid u_{j}<u_{i} ; c_{j}>c_{i}\right\}>\#\left\{j \in S \mid c_{i}<c_{j} \leq k\right\} .
$$

Inequality (2.6) is false, for if $j$ belongs to the set on the left hand side and satisfies $c_{j}>k$, then we have

$$
u_{j}>\bar{u}_{c_{j}} \geq \bar{u}_{k}=u_{i}-1 \text {, }
$$

which is impossible. If on the other hand each index $j$ in this set satisfies $c_{j} \leq k$, then we have the inclusion

$$
\left\{j \in S \mid u_{j}<u_{i} ; c_{j}>c_{i}\right\} \subset\left\{j \in S \mid c_{i}<c_{j} \leq k\right\},
$$

which contradicts the direction of the inequality. We conclude that no element of the set $\{\phi(j) \mid j \in T\}$ is an excedance in $v$, and that we have

$$
E(v)=L(c)=\left\{c_{j} \mid j \in S\right\} .
$$

Finally, we show that $e$ has the defining properties of etab $(v)$. For each index $j$ in $S$, we have defined $e_{c_{j}}=c_{j}$ so that $e$ satisfies condition (1) of Definition 2.1. Let $c^{\prime}$ be the code of $v$. We claim that for each index $i \in T$, we have

$$
e_{\phi(i)}=c_{i}= \begin{cases}\text { the } c_{\phi(i)}^{\prime} \text { th excedance in } v \text { having value at least } u_{i}, & \text { if } c_{\phi(i)}^{\prime}>0, \\ 0, & \text { otherwise }\end{cases}
$$


By our definition of the sequence $\left(d_{i}\right)_{i \in T}$, it suffices to show that $c_{\phi(i)}^{\prime}=d_{i}$ for each index $i$. The subword $v_{\phi(i)+1} \cdots v_{m}$ of $v$ includes $d_{i}$ letters $v_{\phi(j)}$ with $j \in T$ and $v_{\phi(j)}<v_{\phi(i)}$. On the other hand, any excedance in $v$ to the right of $\phi(i)$ has value greater than $v_{\phi(i)}$. We conclude that $c_{\phi(i)}^{\prime}=d_{i}$.

The above procedure inverts $\theta$ because the biword $z$ it produces is the unique rearrangement of $y$ having the desired properties.

Proposition 2.4. Let $v=v_{1} \cdots v_{m}$ be an arbitrary word, and define

$$
z=\left(\begin{array}{l}
v \\
e
\end{array}\right)=\left(\begin{array}{c}
v \\
\operatorname{etab}(v)
\end{array}\right) .
$$

If there is any rearrangement $z^{\prime}$ of $z$ satisfying

$$
z^{\prime}=\left(\begin{array}{c}
v^{\prime} \\
e^{\prime}
\end{array}\right)=\left(\begin{array}{c}
v^{\prime} \\
\operatorname{etab}\left(v^{\prime}\right)
\end{array}\right)
$$

then $z^{\prime}=z$.

Proof. Let $L$ be the letter set of $e$. By Definition 2.1, we must have $E(v)=E\left(v^{\prime}\right)=L$. Let $i$ be an excedance of $v$ and $v^{\prime}$. By condition (1) of Definition 2.1 we must have $e_{i}=e_{i}^{\prime}=i$, and by condition (3) the upper letters $v_{i}$ and $v_{i}^{\prime}$ must be as large as possible. Thus, $\left(z_{i}\right)_{i \in L}=\left(z_{i}^{\prime}\right)_{i \in L}$.

Let $T=[m] \backslash L$ be the set of non-excedance positions of $v$ and $v^{\prime}$, and consider the corresponding subsequences of biletters $\left(z_{i}\right)_{i \in T}$ and $\left(z_{i}^{\prime}\right)_{i \in T}$. By condition (3) of Definition 2.1, the codes of $\left(v_{i}\right)_{i \in T}$ and $\left(v_{i}^{\prime}\right)_{i \in T}$ are determined by the excedances and excedance values in $v$ and $v^{\prime}$. Thus, the two codes must be identical. Applying the argument following Example 2.4, we conclude that $\left(z_{i}\right)_{i \in T}=\left(z_{i}^{\prime}\right)_{i \in T}$.

Combining Propositions 2.3 and 2.4, we complete the proof of Theorem 2.1.

\section{An application of Dumont's statistic}

As an application of Dumont's (generalized) statistic, we will strengthen a special case of a result of Stanley [9, Cor. 4.5] concerning $f$-vectors and $h$-vectors of simplicial complexes.

Given a $(d-1)$-dimensional simplicial complex $\Sigma$, we define its $f$-vector to be

$$
f_{\Sigma}=\left(f_{-1}, f_{0}, f_{1}, \ldots, f_{d-1}\right)
$$

where $f_{i}$ counts the number of $i$-dimensional faces of $\Sigma$. By convention, $f_{-1}=1$. Similarly, we may define the $f$-vector of a poset $P$ by identifying $P$ with its order complex $\Delta(P)$. (See $[10$, p. 120].) That is, we define

$$
f_{P}=f_{\Delta(P)}=\left(f_{-1}, f_{0}, f_{1}, \ldots, f_{d-1}\right),
$$


where $f_{i}$ counts the number of $(i+1)$-element chains of $P$. Again, $f_{-1}=1$ by convention.

In abundant research papers, authors have considered the $f$-vectors of various classes of complexes and posets, and have conjectured or obtained significant information about the coefficients. (See [1], [2], [11, Ch. 2,3].) Such information includes linear relationships between coefficients and properties such as symmetry, log concavity and unimodality.

Related to the $f$-vector $f_{\Sigma}$ is the $h$-vector $h_{\Sigma}=\left(h_{0}, h_{1}, \ldots, h_{d}\right)$, which we define by

$$
\sum_{i=0}^{d} f_{i-1}(x-1)^{d-i}=\sum_{i=0}^{d} h_{i} x^{d-i} .
$$

From this definition, it is clear that knowing the $h$-vector of a complex is equivalent to knowing the $f$-vector. For some conditions on a simplicial complex, one can show that its $h$-vector is the $f$-vector of another complex. Specifically, we have the following result due to Stanley [9, Cor. 4.5].

Theorem 3.1. If $\Sigma$ is a balanced Cohen-Macaulay complex, then its h-vector is the $f$ vector of some simplicial complex $\Gamma$.

We define a simplicial complex to be Cohen-Macaulay if it satisfies a certain topological condition $([11$, p. 61$])$, and balanced if we can color the vertices with $d$ colors such that no face contains two vertices of the same color ([11, p. 95]). The class of balanced CohenMacaulay complexes is quite important because it includes the order complexes of all distributive lattices. The distributive lattices, in turn, contain information about all posets. (See [10, Ch. 3].)

By placing an additional restriction on the complex $\Sigma$, one arrives at a special case of the theorem which has an elegant bijective proof. Let us require that $\Sigma$ be the order complex of a distributive lattice $J(P)$. In this case, $h_{\Sigma}=h_{J(P)}$ counts the number of linear extensions of $P$ by descents. (See [4].) That is, $h_{k}$ is the number of linear extensions of $P$ with $k$ descents. Therefore, Theorem 3.1 asserts that for any poset $P$, there is a bijective correspondence between linear extensions of $P$ with $k$ descents and $(k-1)$-faces of some simplicial complex $\Gamma$.

$$
\{\pi \mid \pi \text { a linear extension of } P \text {; } \operatorname{des}(\pi)=k\} \stackrel{1-1}{\longleftrightarrow}\{\sigma \mid \sigma \text { a }(k-1) \text {-face of } \Gamma\} .
$$

Using [3, Remark 6.6] and [7, Cor. 2.2], one can construct a family $\left\{\Xi_{n}\right\}_{n>0}$ of simplicial complexes such that for any poset $P$ on $n$ elements, the complex $\Gamma$ corresponding to $\Sigma=\Delta(J(P))$ is a subcomplex of $\Xi_{n}$.

On the other hand, any additional restriction placed on the complex $\Sigma$ in Theorem 3.1 should allow us to prove more than a special case of the theorem. It should allow us to strengthen the special case by asserting specific properties of the complex $\Gamma$ in the conclusion of the theorem. In particular, let us require that $\Sigma$ be the order complex of a distributive lattice $J(P)$ which is a product of chains. (See $[10$, Ch. 3] for definitions.) We will prove the following result.

Theorem 3.2. Let the distributive lattice $J(P)$ be a product of chains. Then there is a poset $Q$ such that the h-vector of $J(P)$ is the $f$-vector of $Q$. 


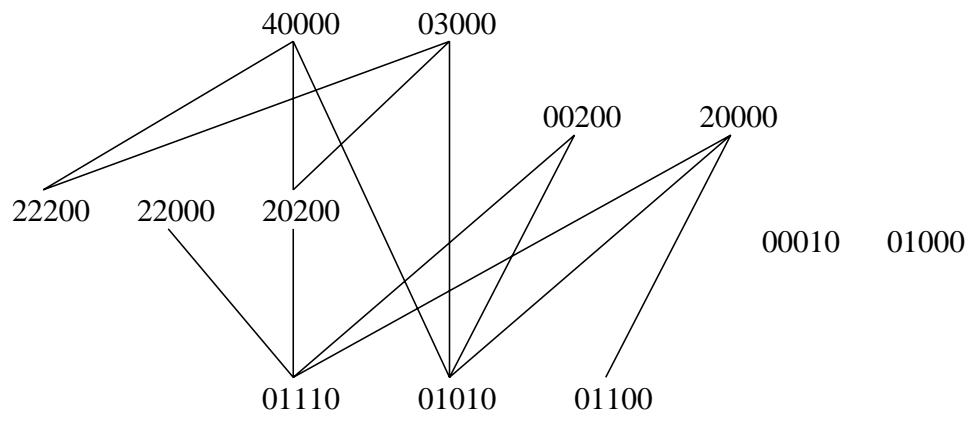

Figure 3.1: A poset with a $k$-element chain for each $k$-letter code in $C(11223)$.

Let us reconsider this theorem in terms of rearrangements of words. If $J(P)$ is a product of chains having cardinalities $\left(p_{1}+1\right), \ldots,\left(p_{n}+1\right)$, then $P$ is the disjoint sum of chains $\left(\mathbf{p}_{\mathbf{1}}+\cdots+\mathbf{p}_{\mathbf{n}}\right)$. It is not difficult to see that linear extensions of $P$ are in bijective correspondence with rearrangements of the word $w=1^{p_{1}} \cdots n^{p_{n}}$. Combining this observation with Theorem 2.1, we restate Theorem 3.2 in terms of Dumont's statistic.

Proposition 3.3. Let $w$ be any word and define the vector $h=\left(h_{0}, \ldots, h_{d}\right)$ by

$$
h_{i}=\#\{u \in R(w) \mid \operatorname{dmc}(u)=i\},
$$

where $d$ is the maximum cardinality of $L C(u)$ over all rearrangements $u$ of $w$. Then, there is a poset $Q$ whose $f$-vector is $h$.

To prove the proposition, and therefore Theorem 3.2, we will work directly with codes of rearrangements of a word. Let us denote $C(w)$ be the set of codes of all rearrangements of $w$. Proposition 3.3 asserts that for any word $w$, there is a bijection between $k$-letter elements of $C(w)$ and $k$-element chains in some poset $Q$,

$$
\{c \in C(w) \mid c \text { a } k \text {-letter code }\} \stackrel{1-1}{\longleftrightarrow}\left\{\left(v_{1}<_{Q} \cdots<_{Q} v_{k}\right) \in \Delta(Q)\right\} .
$$

We will construct such a poset $Q=Q(w)$ as follows.

Definition 3.1. Given an arbitrary word $w$, let $Q$ be the subset of one-letter codes in $C(w)$. For each pair $\left(c, c^{\prime}\right)$ of codes in $Q$ whose letters are $\left(\ell, \ell^{\prime}\right)$, respectively, define $c<_{Q} c^{\prime}$ if

1. $\ell<\ell^{\prime}$.

2. The multiplicity of $\ell$ in $c$ is strictly greater than that of $\ell^{\prime}$ in $c^{\prime}$.

3. For each position $i$ such that $c_{i}^{\prime}=\ell^{\prime}$, we have $c_{i+\ell^{\prime}-\ell}=\ell$.

Example 3.2. Figure 3.1 shows the poset $Q$ corresponding to the word $w=11223$. The $f$-vector $f_{Q}$ counts words in $R(w)$ by Dumont's statistic. Equivalently, it counts linear extensions of the poset $P=\mathbf{2}+\mathbf{2}+\mathbf{1}$ by descent, and is equal to the $h$-vector of $J(P)$,

$$
f_{Q}=h_{J(P)}=(1,12,15,2) .
$$


In Sections 4 and 5 we will demonstrate that for any word $w$, the procedure in Definition 3.1 gives a poset $Q$ satisfying the bijections of (3.1). We will give an explicit bijection $\Psi: C(w) \rightarrow \Delta(Q)$, taking $k$-letter codes in $C(w)$ to $k$-element chains in $Q$.

\section{The chain map $\Psi$}

Fix a nondecreasing word $w=w_{1} \cdots w_{m}$ on $n$ letters, and define the poset $Q$ as in Definition 3.1. We will define a chain map $\Psi: C(w) \rightarrow \Delta(Q)$ which will identify a code $c$ with a chain

$$
\Psi(c)=v_{1}<_{Q} \cdots<_{Q} v_{k}
$$

of elements in $Q$. If $c$ is a code on the $k$ letters $\ell_{1}<\cdots<\ell_{k}$, then each poset element $v_{i}$ will be a code whose unique nonzero letter is $\ell_{i}$. Specifically, we will determine $v_{i}$ by applying a vertex map $\psi_{\ell_{i}}: C(w) \rightarrow Q$ to $c$.

$$
v_{i}=\psi_{\ell_{i}}(c)
$$

After proving that $\psi_{\ell_{i}}(c)<_{Q} \psi_{\ell_{j}}(c)$ whenever $\ell_{i}<\ell_{j}$, we will define the chain map to be a product of vertex maps,

$$
\Psi(c)=\psi_{\ell_{1}}(c)<_{Q} \cdots<_{Q} \psi_{\ell_{k}}(c) .
$$

We begin by observing that several simple operations on codes in $C(w)$ yield other codes in $C(w)$.

Observation 4.1. Let $u$ be a rearrangement of $w$ and let $c=\operatorname{code}(u)$.

1. If $c_{i}>c_{i+1}$, then the word

$$
c^{\prime}=c_{1} \cdots c_{i-1} \cdot c_{i+1} \cdot\left(c_{i}-1\right) \cdot c_{i+2} \cdots c_{m}
$$

belongs to $C(w)$.

2. If for some $r>i, c_{i}$ is strictly less than $c_{i+1}, \ldots, c_{r}$ and $c_{i}>c_{r+1}$, then the word

$$
c^{\prime \prime}=c_{1} \cdots c_{i-1} \cdot c_{r+1} \cdot c_{i+1} \cdots c_{r} \cdot\left(c_{i}-1\right) \cdot c_{r+2} \cdots c_{m}
$$

belongs to $C(w)$.

3. If $c_{i}<c_{i+1}$, or if $c_{i}=c_{i+1}$ and $u_{i}<u_{i+1}$, then the word

$$
c^{\prime \prime \prime}=c_{1} \cdots c_{i-1} \cdot\left(c_{i+1}+1\right) \cdot c_{i} \cdot c_{i+2} \cdots c_{m}
$$

belongs to $C(w)$. 
Proof. Let $u^{\prime}$ be the word obtained from $u$ by switching the letters in positions $i$ and $i+1$, and let $u^{\prime \prime}$ be the word obtained by switching the letters in positions $i$ and $r+1$,

$$
\begin{aligned}
u^{\prime} & =\left(u_{1} \cdots u_{i-1} \cdot u_{i+1} \cdot u_{i} \cdot u_{i+2} \cdots u_{m}\right), \\
u^{\prime \prime} & =\left(u_{1} \cdots u_{i-1} \cdot u_{r+1} \cdot u_{i+1} \cdots u_{r} \cdot u_{i} \cdot u_{r+2} \cdots u_{m}\right) .
\end{aligned}
$$

(1) We have $u_{i}>u_{i+1}$ and $c^{\prime}=\operatorname{code}\left(u^{\prime}\right)$.

(2) We have $u_{r+1}<u_{i}<u_{i+1}, \cdots, u_{r}$ and $c^{\prime \prime}=\operatorname{code}\left(u^{\prime \prime}\right)$.

(3) We have $u_{i}<u_{i+1}$ and $c^{\prime \prime \prime}=\operatorname{code}\left(u^{\prime}\right)$.

Using this observation we will define two families of maps from $C(w)$ to itself, $\lambda_{1}, \ldots, \lambda_{m-1}$ and $\mu_{1}, \ldots, \mu_{m-1}$. Then, composing maps from these two families, we will define the family of vertex maps $\psi_{1}, \ldots, \psi_{m-1}$.

The map $\lambda_{\ell_{i}}: C(w) \rightarrow C(w)$ removes from a code $c$ all letters $\ell_{j}$ which are greater than $\ell_{i}$. It essentially changes each such letter $\ell_{j}$ to $\ell_{i}$ and moves it $\ell_{j}-\ell_{i}$ places to the right in $c$. If we identify $c$ with the $k$-element chain $v_{1}<_{Q} \cdots<_{Q} v_{k}$, then we will identify $\lambda_{\ell_{i}}(c)$ with the $i$-element subchain $v_{1}<_{Q} \cdots<_{Q} v_{i}$.

Definition 4.1. Let $\ell$ be a nonzero letter. Define the map $\lambda_{\ell}: C(w) \rightarrow C(w)$ by performing the following procedure on a code $c$.

For $i=m, m-1, \ldots, 1$, if $c_{i}>\ell$, then

1. Set $\delta=c_{i}-\ell$.

2. Redefine $c=c_{1} \cdots c_{i-1} \cdot c_{i+1} \cdots c_{i+\delta} \cdot \ell \cdot c_{i+\delta+1} \cdots c_{m}$.

Analogous to $\lambda_{\ell_{i}}$, the map $\mu_{\ell_{i}}: C(w) \rightarrow C(w)$ removes all letters which are smaller than $\ell_{i}$. It does so by changing each such smaller letter to 0 . If we identify $c$ with the $k$-element chain $v_{1}<_{Q} \cdots<_{Q} v_{k}$, then we will identify $\mu_{\ell_{i}}(c)$ with the $(k-i+1)$-element subchain $v_{i}<_{Q} \cdots<_{Q} v_{k}$.

Definition 4.2. Let $\ell$ be a nonzero letter. Define the map $\mu_{\ell}: C(w) \rightarrow C(w)$ by $\mu_{\ell}(c)=$ $a_{1} \cdots a_{m}$, where

$$
a_{i}= \begin{cases}0, & \text { if } c_{i}<\ell \\ c_{i}, & \text { otherwise }\end{cases}
$$

The maps $\lambda_{1}, \ldots, \lambda_{m-1}$, and $\mu_{1}, \ldots, \mu_{m-1}$ are well defined, for their definitions are merely repeated applications of Observation 4.1 (1) and (2). Note that the composition $\mu_{\ell} \lambda_{\ell}$ produces a code on the single letter $\ell$. This code is an element of $Q$, and a vertex of $\Delta(Q)$.

Definition 4.3. Let $\ell$ be a nonzero letter. Define the vertex map $\psi_{\ell}: C(w) \rightarrow Q$ by

$$
\psi_{\ell}=\mu_{\ell} \lambda_{\ell}
$$


It is easy to see that $\lambda_{\ell}^{2}=\lambda_{\ell}$, and therefore that $\psi_{\ell} \lambda_{\ell}=\psi_{\ell}$. These and the following relations will be essential in establishing a bijection between $C(w)$ and $\Delta(Q)$.

Proposition 4.2. Let $\ell$ and $\ell^{\prime}$ be letters, $1 \leq \ell<\ell^{\prime} \leq n$. The maps $\lambda_{\ell}, \lambda_{\ell^{\prime}}, \psi_{\ell}$, and $\psi_{\ell^{\prime}}$ satisfy the relations

1. $\lambda_{\ell^{\prime}} \lambda_{\ell}=\lambda_{\ell} \lambda_{\ell^{\prime}}=\lambda_{\ell}$

2. $\psi_{\ell} \lambda_{\ell^{\prime}}=\psi_{\ell}$

3. $\psi_{\ell}(c)<_{Q} \psi_{\ell^{\prime}}(c)$, if c contains both letters.

Proof. (1) Let $c=\operatorname{code}(u)$ be an element of $C(w)$. By the comments following Definition 4.2 , we may interpret $\lambda_{\ell}(c)$ as follows. Define $b=b_{1} \cdots b_{m}$ by

$$
b_{i}= \begin{cases}\ell, & \text { if } c_{i}>\ell \\ c_{i}, & \text { otherwise }\end{cases}
$$

and rearrange the biword $\left(\begin{array}{l}u \\ b\end{array}\right)$ as $\left(\begin{array}{l}u^{\prime} \\ b^{\prime}\end{array}\right)$ so that $b^{\prime}=\operatorname{code}\left(u^{\prime}\right)$. Then, $b^{\prime}=\lambda_{\ell}(c)$.

It is not hard to see that there is a unique such rearrangement. Using this interpretation, it is easy to see that $\lambda_{\ell^{\prime}} \lambda_{\ell}, \lambda_{\ell} \lambda_{\ell^{\prime}}$, and $\lambda_{\ell}$ describe the same procedure.

(2) Using (1), we have $\psi_{\ell} \lambda_{\ell^{\prime}}=\mu_{\ell} \lambda_{\ell} \lambda_{\ell^{\prime}}=\mu_{\ell} \lambda_{\ell}=\psi_{\ell}$.

(3) We may assume that $\ell^{\prime}$ is the greatest letter in $c$. (Otherwise, we define $d=\lambda_{\ell^{\prime}}(c)$ and note that $\psi_{\ell}(c)=\psi_{\ell}(d)$ and $\psi_{\ell^{\prime}}(c)=\psi_{\ell^{\prime}}(d)$.) Let $e=\psi_{\ell}(c)$ and $e^{\prime}=\psi_{\ell^{\prime}}(c)$. Clearly, the multiplicity of $\ell$ in $e$ is strictly greater than that of $\ell^{\prime}$ in $e^{\prime}$, for

$$
\#\left\{i \mid e_{i}=\ell\right\}=\#\left\{i \mid c_{i} \geq \ell\right\}>\#\left\{i \mid c_{i} \geq \ell^{\prime}\right\}=\#\left\{i \mid e_{i}^{\prime}=\ell^{\prime}\right\}
$$

Next, we show that for any position $i$ of $e^{\prime}$ satisfying $e_{i}^{\prime}=\ell$, we must have $e_{i+\ell^{\prime}-\ell}=\ell$. Since by assumption, $\ell^{\prime}$ is the greatest letter in $c$, we have $e_{i}^{\prime}=\ell^{\prime}$ if and only if $c_{i}=\ell^{\prime}$. To find $e$, we first calculate $\lambda_{\ell}(c)$ by the procedure of Definition 4.1. At each iteration $i$ such that $c_{i}=\ell^{\prime}$, we place the letter $\ell$ into position $i+\ell^{\prime}-\ell$ of $\lambda_{\ell}(c)$. This position will not be altered by iterations $i-1, \ldots, 1$, since all letters of $c$ are no greater than $\ell^{\prime}$. Finally, since $e=\mu_{\ell} \lambda_{\ell}(c)$, and $\mu_{\ell}$ changes only those letters less than $\ell$, we see that $e_{i+\ell^{\prime}-\ell}=\ell$ for every position $i$ such that $e_{i}=\ell^{\prime}$.

Now we may define the map $\Psi$.

Definition 4.4. Define the chain map $\Psi: C(w) \rightarrow \Delta(Q)$ by

$$
\Psi(c)=\psi_{\ell_{1}}(c)<_{Q} \cdots<_{Q} \psi_{\ell_{k}}(c),
$$

where $\ell_{1}<\cdots<\ell_{k}$ are the distinct nonzero letters in $c$. 


\section{$5 \quad$ Inverting $\Psi$}

We will define a map $\Phi: \Delta(Q) \rightarrow C(w)$ which takes a $k$-element chain in $Q$ to a $k$ letter code in $C(w)$. By demonstrating that $\Phi$ inverts $\Psi$, we will complete the proof of Proposition 3.3.

We begin by defining an operation $\vee$ on a subset of $C(w) \times Q$. This operation joins a new letter to a code.

Definition 5.1. Let $d \in Q$ be a code whose unique nonzero letter is $\ell^{\prime}$, and let $c \in C(w)$ be a code whose greatest letter is $\ell$ and which satisfies $\psi_{\ell}(c)<_{Q} d$. Let $\delta=\ell^{\prime}-\ell$ and define the code $e=c \vee d$ by the following procedure.

1. For each $i$ such that $d_{i}=\ell^{\prime}$, set $e_{i}=\ell^{\prime}$ and cross out the $\ell$ in position $i+\delta$ of $c$.

2. Fill the remaining positions of $e$ with the remaining components of $c$, in order.

Note that $L(e)=L(c) \cup\{\ell\}$. Therefore, we may map a chain of $k$ one-letter codes to a single $k$-letter code by iterating the join operation.

Definition 5.2. Let $v_{1}<_{Q} \cdots<_{Q} v_{k}$ be a chain of one-letter codes on the letters $\ell_{1}<$ $\cdots<\ell_{k}$, respectively. Define the map $\Phi: \Delta(Q) \rightarrow C(w)$ by

$$
\Phi\left(v_{1}<_{Q} \cdots<_{Q} v_{k}\right)=\left(\cdots\left(\left(v_{1} \vee v_{2}\right) \vee v_{3}\right) \cdots\right) \vee v_{k}
$$

The following proposition shows that the join operation is well defined. It follows that $\Phi$ is well defined also.

Proposition 5.1. If $c$ and $d$ are codes in $C(w)$ satisfying the hypotheses of Definition 5.1, then $c \vee d$ also belongs to $C(w)$.

Proof. Let $u$ and $y$ be words in $R(w)$ whose codes $c=\operatorname{code}(u)$ and $d=\operatorname{code}(y)$ satisfy the conditions of Definition 5.1. Consider the leftmost position $i$ in $c$ such that $c_{i}=\ell$ and $d_{i-\delta}=\ell^{\prime}$, where $\delta=\ell^{\prime}-\ell$. By assumption, $c_{i-1} \leq c_{i}$. If $c_{i-1}<c_{i}$, or if $c_{i-1}=c_{i}$ and $u_{i-1}<u_{i}$, then we may apply Observation 4.1 (3) $\ell^{\prime}-\ell$ times to obtain the word

$$
c_{1} \cdots c_{i-\delta-1} \cdot \ell^{\prime} \cdot c_{i-\delta} \cdots \hat{c}_{i} \cdots c_{m}
$$

which belongs to $C(w)$. (Here, $\hat{c}_{i}$ means that the letter $c_{i}$ is omitted.) Repeating this process for each such position $i$, we redefine the join operation. Therefore it suffices to show that for every position $i$ satisfying $d_{i-\delta-1}=0, d_{i-\delta}=\ell^{\prime}$, and $c_{i-1}=c_{i}=\ell$, we have $u_{i-1}<u_{i}$.

Let $i$ be such a position and suppose that $u_{i-1}=u_{i}$. Since $d_{i-\delta}=\ell^{\prime}$ and $d_{i-\delta-1}=0$, there are exactly $i-\delta-1+\ell^{\prime}=i+\ell-1$ letters in $y$ which are strictly less than $y_{i-\delta}$. In particular we have

$$
\bar{w}_{i+\ell-1}<\bar{w}_{i+\ell}
$$


Let $k$ be the number of positions preceding $i$ such that $u_{i-k}=u_{i-k+1}=\cdots=u_{i}$ and $c_{i-k}=c_{i-k+1}=\cdots=\ell$. Then there are exactly $i-k-1+\ell$ letters in $u$ which are strictly less than $u_{i}\left(=u_{i-1}=\cdots=u_{i-k}\right)$. In particular we have

$$
\bar{w}_{i-k-1+\ell}<\bar{w}_{i-k+\ell}=\bar{w}_{i-k+1+\ell}=\cdots=\bar{w}_{i+\ell}
$$

which contradicts (5.1). We conclude that $u_{i-1}<u_{i}$, and therefore that $c \vee d$ belongs to $C(w)$.

The following identities relate the maps $\psi$ and $\lambda$ to the join operation $\vee$.

Proposition 5.2. The pair of maps $(\psi, \lambda)$ inverts the operation $\vee$ in the following sense.

1. Let $c \in C(w)$ be a code with greatest letter $\ell$, and let $d \in Q$ be a code with letter $\ell^{\prime}>\ell$ and satisfying $\psi_{\ell}(c)<_{Q} d$. Then we have

$$
\begin{aligned}
\psi_{\ell^{\prime}}(c \vee d) & =d, \\
\lambda_{\ell}(c \vee d) & =c .
\end{aligned}
$$

2. Let $c \in C(w)$ be a code whose greatest two letters are $\ell<\ell^{\prime}$. Then we have

$$
\lambda_{\ell}(c) \vee \psi_{\ell^{\prime}}(c)=c .
$$

Proof. (1) Let $S$ be the set of positions of $d$ containing the letter $\ell^{\prime}$, and let $\delta=\ell^{\prime}-\ell$.

Define the words $e=c \vee d, d^{\prime}=\psi_{\ell^{\prime}}(c \vee d)$, and $c^{\prime}=\lambda_{\ell}(c \vee d)$. Calculating $e$, we have

$$
\begin{aligned}
& \left(e_{i}\right)_{i \in S}=\ell^{\prime} \cdots \ell^{\prime}, \\
& \left(e_{i}\right)_{i \notin S}=\left(c_{i}\right)_{i-\delta \notin S} .
\end{aligned}
$$

Since $e$ contains no letters greater than $\ell^{\prime}$, we have $d^{\prime}=\psi_{\ell^{\prime}}(e)=\mu_{\ell^{\prime}}(e)$. Thus, $d^{\prime}=d$ :

$$
\begin{aligned}
& \left(d_{i}^{\prime}\right)_{i \in S}=\ell^{\prime} \cdots \ell^{\prime}, \\
& \left(d_{i}^{\prime}\right)_{i \notin S}=0 \cdots 0 .
\end{aligned}
$$

Calculating $c^{\prime}=\lambda_{\ell}(e)$, we change each occurrence of $\ell^{\prime}$ in $e$ to $\ell$, and move it $\delta$ positions to the right. Since $\psi_{\ell}(c)<_{Q} d$, we see that $c^{\prime}=c$ :

$$
\begin{aligned}
& \left(c_{i}^{\prime}\right)_{i-\delta \in S}=\ell \cdots \ell=\left(c_{i}\right)_{i-\delta \in S}, \\
& \left(c_{i}^{\prime}\right)_{i-\delta \notin S}=\left(c_{i}\right)_{i-\delta \notin S} .
\end{aligned}
$$

(2) Similar.

Finally, we demonstrate that $\Phi$ inverts $\Psi$.

Proposition 5.3. Let $c \in C(w)$ be a code on the letters $\ell_{1}<\cdots<\ell_{k}$, and let $v_{1}<_{Q}$ $\cdots<_{Q} v_{k}$ be a k-element chain in $Q$, where the letter of $v_{i}$ is $\ell_{i}$ for each $i$. The maps $\Psi$ and $\Phi$ satisfy 
1. $\Psi \Phi\left(v_{1}<_{Q} \cdots<_{Q} v_{k}\right)=v_{1}<_{Q} \cdots<_{Q} v_{k}$.

2. $\Phi \Psi(c)=c$.

Proof. (1) By Definition 5.2, we have

$$
\Phi\left(v_{1}<_{Q} \cdots<_{Q} v_{k}\right)=\left(\cdots\left(\left(v_{1} \vee v_{2}\right) \vee v_{3}\right) \cdots\right) \vee v_{k}
$$

Applying $\Psi=\psi_{\ell_{1}} \times \cdots \times \psi_{\ell_{k}}$ to this code, we calculate $\psi_{\ell_{i}}\left(\left(\cdots\left(v_{1} \vee v_{2}\right) \vee \cdots\right) \vee v_{k}\right)$, for $i=1, \ldots, k$. By Proposition $5.2(1)$, we have

$$
\begin{aligned}
\psi_{\ell_{i}}\left(\left(\cdots\left(v_{1} \vee v_{2}\right) \vee \cdots\right) \vee v_{k}\right) & =\psi_{\ell_{i}} \lambda_{\ell_{i}} \lambda_{\ell_{i+1}} \cdots \lambda_{\ell_{k}}\left(\left(\cdots\left(v_{1} \vee v_{2}\right) \vee \cdots\right) \vee v_{k}\right) \\
& =\psi_{\ell_{i}}\left(\left(\cdots\left(v_{1} \vee v_{2}\right) \vee \cdots\right) \vee v_{i}\right) \\
& =v_{i}
\end{aligned}
$$

as desired.

(2) By Definition 4.4, we have

$$
\Psi(c)=\psi_{\ell_{1}}(c)<_{Q} \cdots<_{Q} \psi_{\ell_{k}}(c)
$$

Applying $\Phi$ to this chain, we join vertices one at a time. Noting that $\psi_{\ell_{1}}(c)=\lambda_{\ell_{1}}(c)$, we use Proposition 5.2 (2) to calculate

$$
\begin{aligned}
\lambda_{\ell_{i}}(c) \vee \psi_{\ell_{i+1}}(c) & =\lambda_{\ell_{i}} \lambda_{\ell_{i+1}}(c) \vee \psi_{\ell_{i+1}} \lambda_{\ell_{i+1}}(c) \\
& =\lambda_{\ell_{i}}\left(\lambda_{\ell_{i+1}}(c)\right) \vee \psi_{\ell_{i+1}}\left(\lambda_{\ell_{i+1}}(c)\right) \\
& =\lambda_{\ell_{i+1}}(c) .
\end{aligned}
$$

Thus, after $k-1$ join iterations, we recover $c$.

This completes the proof of Theorem 3.2.

\section{Open questions}

Since the class of balanced Cohen-Macaulay complexes contains so many widely studied classes of complexes, there are many possibilities to refine Theorem 3.1. In Theorem 3.2, we have required that $\Sigma$ be an order complex of the form $\Delta(J(P))$, where $P$ is a disjoint sum of chains. One could also ask if the theorem holds for more general classes of posets. (See [10], [11] for definitions in the questions that follow.) For instance, the following questions are open.

Question 6.1. If $P$ is a forest, then is there another poset $Q$ such that the $h$-vector of $J(P)$ is the $f$-vector of $Q$ ?

Question 6.2. If $P$ is a series-parallel poset, then is there another poset $Q$ such that the $h$-vector of $J(P)$ is the $f$-vector of $Q$ ? 
We conjecture that the answers to both questions are affirmative. In fact, we conjecture that the answer remains affirmative for any choice of a poset $P$.

Conjecture 6.1. Let $J(P)$ be any distributive lattice. Then there is another poset $Q$ such that the h-vector of $J(P)$ is the $f$-vector of $Q$.

This conjecture has been tested by computer for all distributive lattices $J(P)$ arising from posets $P$ having up to seven elements. Other open questions place requirements on $\Gamma$ instead of on $\Sigma$.

Question 6.3. For which balanced Cohen-Macaulay complexes $\Sigma$ is $h_{\Sigma}$ the $f$-vector of a graded poset (or $(\mathbf{3}+\mathbf{1})$-free poset, or flag complex)?

To begin to answer Questions 6.1 - 6.3, it would be interesting to utilize any Eulerian permutation statistic stat to define posets such as $Q$ in Definition 3.1 which satisfy the following two conditions.

1. For each $k$, the $k$-element chains in $Q$ bijectively correspond to the linear extensions $\pi$ of $P$ with $\operatorname{stat}(\pi)=k$.

2. For each poset $P$ in some class $\mathcal{P}$, the statistics stat and des are equidistributed on the set of linear extensions of $P$, so that $h_{J(P)}=f_{Q}$.

One might also consider a variation of this method based upon objects other than permutations, such as Motzkin paths or either of the tree representations in [10, pp. 23-25].

A result similar to Theorem 2.1 (in the sense that word rearrangements correspond to linear extensions of certain posets) states that the statistics INV and MAJ are equally distributed on the linear extensions of posets known as postorder labelled forests [5]. Perhaps Theorem 2.1 could be extended similarly.

Question 6.4. For what conditions on a poset $P$ are the statistics des and dmc equidistributed on the set of linear extensions of $P$ ?

One might apply another variation of the method above by defining a rule which maps each $n$-element poset $P$ to a subset $\mathcal{K}(P)$ of $S_{n}$ which is not a set of linear extensions of $P$. This subset should have the property that the elements $\pi$ in $\mathcal{K}(P)$ satisfying $\operatorname{stat}(\pi)=k$ are in bijective correspondence with the linear extensions of $P$ which have $k$ descents.

\section{Acknowledgments}

Conversations with Einar Steingrímsson, Richard Stanley, Dominique Dumont, and Dominique Foata aided greatly in the writing of this paper. Referees from the Electronic Journal of Combinatorics were very helpful as well. In particular their suggestions led to an improved proof of Proposition 2.3. 


\section{References}

[1] M. M. BAyer AND L. J. BillerA, Counting faces and chains in polytopes and posets, Contemp. Math., 34 (1984), pp. 207-252.

[2] L. J. Billera AND A. BJÖRner, Face numbers of polytopes and complexes, in Handbook of Discrete and Computational Geometry, J. E. Goodman and J. O’Rourke, eds., CRC Press, Boca Raton/New York, 1997, pp. 291-310.

[3] A. Björner, P. Frankl, And R. Stanley, The number of faces of balanced Cohen-Macaulay complexes and a generalized Macaulay theorem, Combinatorica, 7 (1987), pp. 23-34.

[4] A. Björner, A. Garsia, And R. Stanley, An introduction to the theory of Cohen-Macaulay posets, in Ordered Sets, I. Rival, ed., Reidel, Dordrecht/Boston/London, 1982, pp. 583-615.

[5] A. BjÖrner And M. Wachs, Permutation Statistics and Linear Extensions of Posets, J. Combin. Theory Ser. A, 58 (1991), pp. 85-114.

[6] D. Dumont, Interprétations combinatoires des nombres de Genocchi, Duke Math. J., 41 (1974), pp. 305-318.

[7] P. Edelman And V. Reiner, H-shellings and h-complexes, Adv. Math., 106 (1994), pp. 36-64.

[8] D. FoATA, Distributions Eulériennes et Mahoniennes sur le groupe des permutations, in Higher Combinatorics, M. Aigner, ed., vol. 19, D. Reidel Publishing Company, Dordrecht-Holland, 1977, pp. 27-49.

[9] R. Stanley, Balanced Cohen-Macaulay complexes, Trans. of the AMS, 241 (1979), pp. 139-157.

[10] - Enumerative Combinatorics, vol. 1, Wadsworth \& Brooks/Cole, Belmont, CA, 1986.

[11] — Combinatorics and Commutative Algebra, Birkhäuser, Boston, MA, 1996. 